\title{
Erratum: Education hubs and private higher education expansion in small island developing states contexts: The case of Mauritius
}

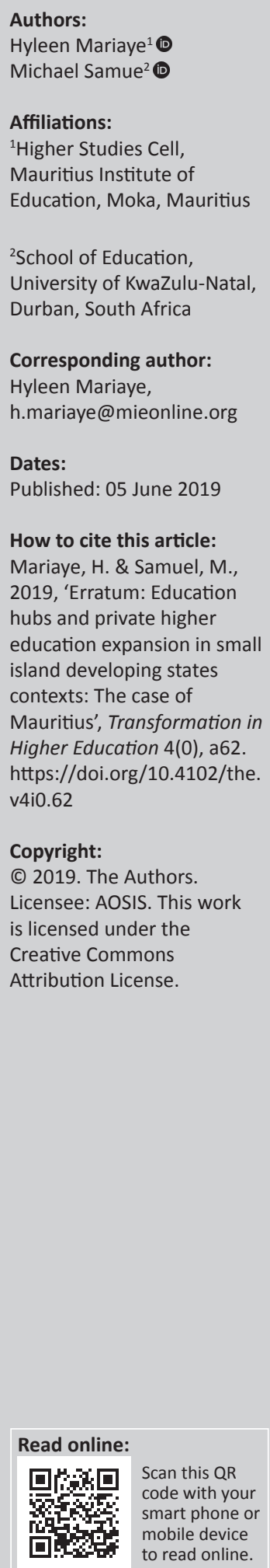

This article published in volume 3 was incorrectly categorised as a Review Article. The article category is hereby corrected as Original Research. This correction does not alter the study's findings of significance or overall interpretation of the study results. The editor apologises for any inconvenience caused. 